\title{
Novel Thin and Compact H-Plane SIW Horn Antenna
}

\author{
Marc Esquius-Morote, Student Member, IEEE, Benjamin Fuchs, Member, IEEE, Jean-François Zürcher, and \\ Juan R. Mosig, Fellow, IEEE
}

\begin{abstract}
The substrate integrated waveguide (SIW) technology allows to construct several types of commonly used antennas in a planar way. However, some practical constraints limit their performances when frequencies below $20 \mathrm{GHz}$ are considered. In the case of SIW horn antennas, the available substrates are much thinner than the wavelength yielding to poor matching and undesired back radiation. In this paper, an innovative structure to overcome these limitations is presented. It consists of a transition printed on the same SIW substrate, which improves both the radiation and the matching performances of conventional SIW horns. The horn shape is also further optimized by reducing its dimensions required for a given directivity. This is obtained by modifying the horn profile in order to effectively combine different TE modes. Guidelines are provided to design this type of thin and compact SIW horn antenna. They were applied to manufacture a prototype in the $\mathrm{Ku}$-band with a substrate thinner than $\lambda_{0} / 10$. Measurement results validate the proposed concepts showing excellent performances.
\end{abstract}

Index Terms-End-fire, front-to-back ratio, horn antenna, horn taper, low profile, printed transition, size reduction, substrate integrated waveguide, vertical polarization.

\section{INTRODUCTION}

$\mathbf{T}$ HE substrate integrated waveguide (SIW) technology is a very promising candidate specially for systems operating in the millimeter-wave region. The most significant advantage of SIW is the possibility to integrate all the components on the same substrate, including passive components (filters, couplers, etc.), active elements (oscillators, amplifiers, etc.) as well as antennas [1]-[7]. This allows the possibility to create substrate integrated circuits (SICs).

For applications involving SICs that require vertically polarized signals radiating at the end-fire, the most commonly used antenna is a H-plane aperture or horn [8]-[11]. However, the performances of this kind of antennas diminish significantly when the substrate thickness $h$ is much smaller than the free space wavelength $\lambda_{0}$ [12], [13]. In general, for thicknesses $h$ smaller than $\lambda_{0} / 6$, the mismatch between the antenna aperture and the air results in unwanted radiation and poor matching.

Manuscript received October 23, 2012; revised January 18, 2013; accepted March 09, 2013. Date of publication March 22, 2013; date of current version May 29, 2013. This work was supported by the Swiss Science and Technology Federal Department of Defense, Civil Protection and Sport (DDPS, ArmaSuisse) under the contract $\mathrm{Nr}$ 041-18.

The authors are with the Laboratoire d'Electromagnétisme et d'Acoustique (LEMA), Ecole Polytechnique Fédérale de Lausanne (EPFL), CH-1015 Lausanne, Switzerland (e-mail: marc.esquiusmorote@epfl.ch).

Color versions of one or more of the figures in this paper are available online at http://ieeexplore.ieee.org.

Digital Object Identifier 10.1109/TAP.2013.2254449

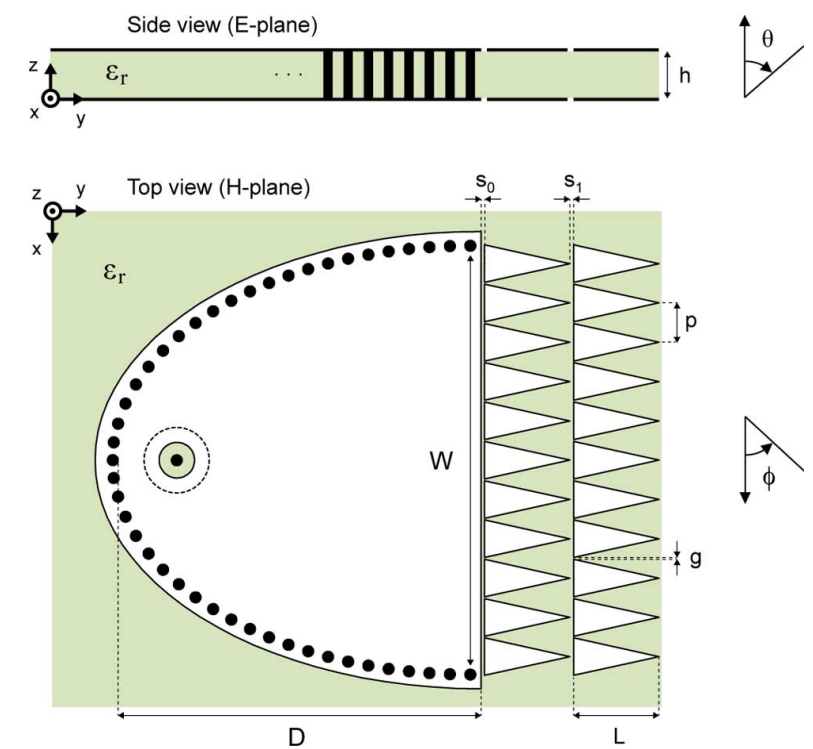

Fig. 1. Side and top view of the proposed thin and compact SIW horn. Metal is represented in white, the dielectric substrate in grey and the metallized via holes in black.

This situation is frequently encountered at frequencies lower than $20 \mathrm{GHz}$. At this frequency range, most commonly available substrates are indeed thinner than $\lambda_{0} / 6$. Furthermore, the existing thicker substrates $(h>2.5 \mathrm{~mm})$ are not suitable for manufacturing SIW components because the via-hole metalization is then challenging and eventually flawed. For these reasons, the SIW technology is mainly used in the millimeter-wave region.

Aiming to extend the use of SIW horn antennas below $20 \mathrm{GHz}$, we have proposed recently [13] an integrated solution to improve their reflection coefficient $\left|S_{11}\right|$ even when built in very thin substrates, i.e., $h<\lambda_{0} / 10$. This solution is based on a printed transition etched after the horn aperture and does not require the use of external bulky elements. Thus, the most important features of the SIW technology, namely its compactness and ease of manufacturing, are maintained.

In this paper, this transition is used as starting point to design a new thin SIW horn antenna with radiation improvements. In particular, the transition is adapted to improve, on top of the matching, the front-to-back ratio (FTBR). A sketch of the proposed SIW horn antenna is shown in Fig. 1.

The paper is organized as follows. In Section II, the main radiation characteristics of horn antennas and known integrated solutions to improve their performances are reviewed. The feasibility of these solutions when using thin substrates is also discussed. In Section III, the radiation properties of a SIW horn antenna with the printed transition developed in [13] are studied 
and a way to improve the FTBR is proposed. The conditions to improve the FTBR and the matching are compared in Section IV and a strategy to achieve both improvements within the same frequency range is presented.

At this point, a thin SIW horn with high FTBR $(>15 \mathrm{~dB})$ and good matching $\left(\left|S_{11}\right|<-10 \mathrm{~dB}\right)$ can be already designed. As a final improvement, a more compact and efficient horn taper is proposed in Section V. Finally, the steps to design a thin and compact SIW horn are described in Section VI and used to manufacture a prototype which confirms the theoretical and simulated values.

\section{SIW HORN RADIATION CHARACTERISTICS}

H-plane horn antennas are created by flaring the dimension of a rectangular waveguide parallel to the $\mathrm{H}$-field, while keeping the perpendicular dimension constant. Thus, the radiation is focused in the H-plane and, as the flare angle is increased, the $\mathrm{H}$-plane beamwidth becomes narrower up to a given flare. Beyond that point, the pattern starts to broaden, due essentially to the phase error across the aperture of the horn [14].

The design rules for SIW H-plane horn antennas follow the same principles as for conventional horns and, therefore, the same strategies to improve their performances can be used. However, the constraints associated with the SIW technology described in Section I make some solutions more feasible than others.

A common way to improve the horn performances and to reduce its dimensions is to place a lens over the horn aperture. Usually, dielectric lenses are used since metal-plate lenses introduce a polarizing effect and their edges cause diffraction. In the case of SIW horns, this solution is easy to implement as the lens can be created by extending the same dielectric slab where the horn is built [9], [10]. These horns are known as lens-corrected horns [15], where the lens focuses the radiation frontwards increasing the FTBR and reducing the phase error. This solution provides good performances in terms of FTBR $(\sim 15 \mathrm{~dB})$ as reported in [10]. Nevertheless, for $h<\lambda_{0} / 6$, this approach is not feasible, because the effect of the lens is then negligible.

To illustrate this limitation, the commercial full wave electromagnetic solver Ansys HFSS v13 was used to simulate the H-plane radiation patterns of a lens-corrected SIW horn with different substrate thicknesses $h$ (see Fig. 2). The substrate permittivity is $\varepsilon_{r}=4.5$ and the horn dimensions are shown in the inset of Fig. 2. As $h$ is reduced, the effect of the lens becomes less relevant: the beamwidth is increased (i.e., no phase correction) and the FTBR is decreased (i.e., no focusing). As expected, for a $h<\lambda_{0} / 10$ the SIW horn radiation resembles the one of a slot antenna with a low FTBR. Notice that $\phi$-angles are defined in such a way that the horn front direction is $\phi=90^{\circ}$ and the back direction $\phi=270^{\circ}$.

The phase error is not a major problem because it can be solved by increasing the horn H-plane dimensions. However, the FTBR remains an important issue since the E-plane dimension $h$ cannot be increased.

Another solution which aims to improve the radiation performances of end-fire SIW antennas has been presented in [11]. In this case, the antenna directivity is increased by placing two directors after the horn aperture which focus the radiation at the

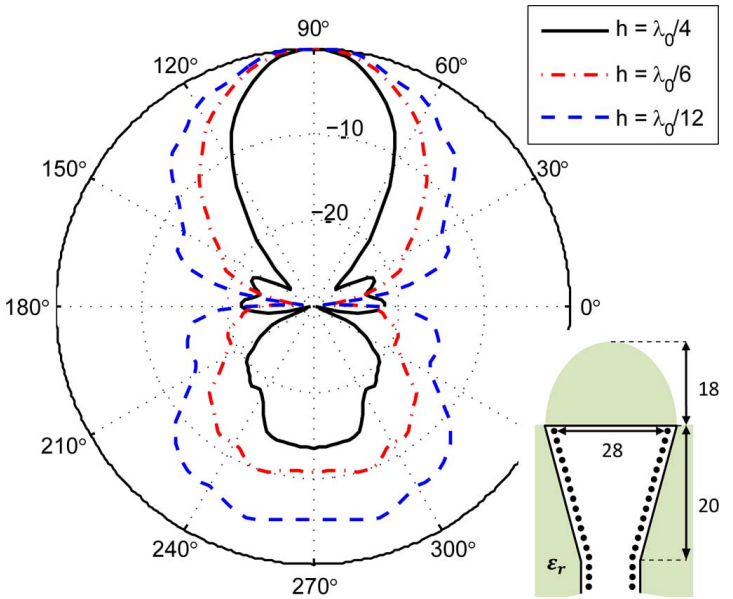

Fig. 2. Simulated normalized radiation patterns (H-plane) at $16 \mathrm{GHz}$ of a lens corrected SIW horn antenna in a substrate of different thicknesses. The dimensions of the horn are in $\mathrm{mm}$ and $\varepsilon_{r}=4.5$.

E-plane. Nevertheless, a substrate of $h \sim \lambda_{0} / 5$ is used and, if $h$ is reduced, the radiation and matching performances degrade akin to the previous example.

The main goal of this paper is to propose an integrated solution which keeps good radiation performances even in case of thin substrates with thickness $h<\lambda_{0} / 10$.

\section{Proposed Integrated Solution for FTBR IMPROVEMENTS}

The printed transition presented in [13] and shown in Fig. 3(a) was specifically designed to improve the matching of thin SIW horns. This transition is etched after the horn aperture and can be seen as a concatenation of blocks, each one being a parallel plate waveguide of length $L_{i}$ separated by gaps of width $s_{i}$. While this transition effectively improves the $\left|S_{11}\right|$ performances, it does not practically alter its radiation characteristics thus, resulting into a low FTBR, typically below $5 \mathrm{~dB}$ [13].

In Sections IV-VII, the transition is revisited from the radiation point of view and modified to improve the FTBR.

\section{A. Array Analogy}

It is known that the radiation of a H-plane horn antenna mainly comes from the edges of the aperture that are parallel to the H-plane. In the case of the SIW horn with a 2 block transition represented in Fig. 3(a), there are three apertures in the $X Z$ plane contributing to the radiation: $E_{0}, E_{1}$ and $E_{2}$ (see Fig. 3(b)). These apertures can be seen as radiating elements of an array along the $y$ axis. Then, by properly choosing the amplitudes and phases of these elements, the radiation pattern and consequently the FTBR of the SIW horn can be improved.

The gap $s_{i}$ affects the amplitudes $A_{i}$ and $A_{i+1}$ of the elements $E_{i}$ and $E_{i+1}$ : when $s_{i}$ becomes larger, $E_{i}$ radiates better, i.e., $A_{i}$ increases, but less energy arrives to the next element, i.e., $A_{i+1}$ decreases. There is a trade-off between no radiation (small $s_{i}$ ) and energy leakage (large $s_{i}$ ). For instance, in our case, if $s_{0}$ is too big then $A_{0} \gg A_{1}, A_{2}$ and the antenna radiation will mainly depend on the element $E_{0}$. Another extreme case would be when $s_{0}$ and $s_{1}$ are too small making then $A_{0}, A_{1} \ll A_{2}$ hence being $E_{2}$ the only radiating element. 


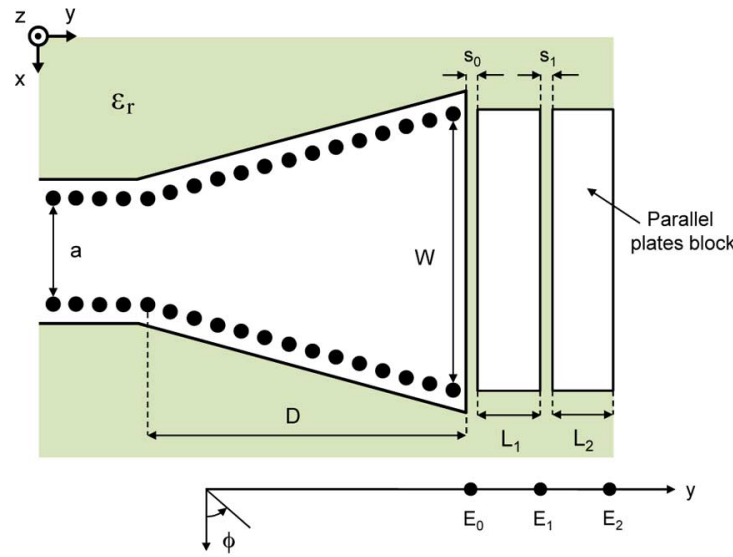

(a)

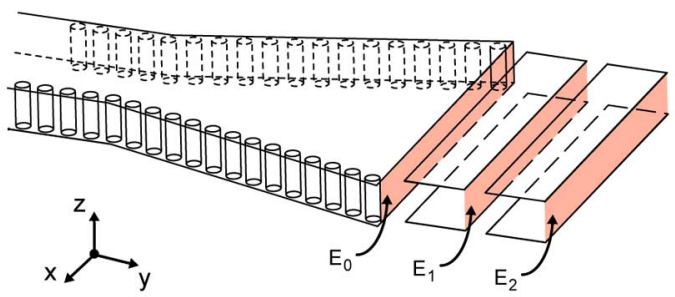

(b)

Fig. 3. H-plane SIW horn with a 2 block printed transition to the air. (a) Top view with the associated notations. (b) $3 \mathrm{D}$ view showing the associated radiating apertures (in red) that constitute the elements of the array analogy.

The phase difference $\alpha_{i, i+1}$ between two adjacent elements $E_{i}$ and $E_{i+1}$ is controlled by the length $L_{i+1}$ that separates both elements. Indeed, $\alpha_{i, i+1} \simeq \beta_{p p} L_{i+1}$ where $\beta_{p p}=2 \pi \sqrt{\varepsilon_{r p p}} / \lambda_{0}$ is the propagation constant of the parallel plate waveguide. An accurate value for the effective permittivity $\varepsilon_{r p p}$ can be obtained by using the quasi-static approximation and figures presented in [16].

The radiation pattern $f(\phi)$ of the SIW horn of Fig. 3(a) can then be expressed using standard array theory as:

$$
\begin{array}{rl}
f(\phi) \simeq A_{0} & g(\phi)+A_{1} e^{j \alpha_{01}} g(\phi) e^{-j\left(2 \pi / \lambda_{0}\right) L_{1} \sin (\phi)} \\
& +A_{2} e^{j\left(\alpha_{01}+\alpha_{12}\right)} g(\phi) e^{-j\left(2 \pi / \lambda_{0}\right)\left(L_{1}+L_{2}\right) \sin (\phi)}
\end{array}
$$

where $g(\phi)$ denotes the radiation pattern of one element and it has been assumed that $L_{i} \gg s_{i}$.

Let us discuss the choice of $s_{i}$ that controls $A_{i}$ and $A_{i+1}$. It is explained in [13] that at least one gap $s_{i}$ should be much smaller than $\lambda_{0}$ to have a good matching at one resonant frequency. At the same time, two elements are required to have an array and thus to improve the FTBR. Therefore, since the SIW horn of Fig. 3(a) has 2 gaps, one can choose the gap $s_{0}$ to be much smaller than $\lambda_{0}$ for matching purposes (which also implies $A_{0} \ll A_{1}, A_{2}$ ) and then use the gap $s_{1}$ to control $A_{1}$ and $A_{2}$ as desired. Hence, the radiation pattern is mainly defined by the elements $E_{1}$ and $E_{2}$.

Regarding the value of $L_{i}$, for the sake of simplicity, the same strip length is chosen for both blocks, i.e., $L_{1}=L_{2}=L$, which yields $\alpha_{01}=\alpha_{12}=\alpha$. The effect of $L$ in the matching performances will be discussed in Section IV.
In this case, the radiation pattern $f(\phi)$ of the SIW horn with a 2 block transition becomes:

$$
f(\phi) \simeq\left(A_{1}+A_{2} e^{j \alpha} e^{-j\left(2 \pi L / \lambda_{0}\right) \sin (\phi)}\right) g(\phi) .
$$

Then, assuming that $s_{1}$ has been chosen such as $A_{1} \simeq A_{2}$, the relation found to minimize $f(-\pi / 2)$ and that also maximizes the FTBR is:

$$
\frac{2 \pi L}{\lambda_{0}}+\beta_{p p} L=(2 n+1) \pi, \text { with } n \in \mathbb{Z} .
$$

Finally, the shortest length $L$ that fulfills (3) thus maximizing the FTBR of the SIW horn at the frequency $f_{F T B R}$ is:

$$
L=\frac{c}{2 f_{F T B R}\left(1+\sqrt{\varepsilon_{r p p}}\right)}
$$

where $c$ denotes the speed of light in free space.

\section{B. Validation of the Array Analogy}

To validate the array analogy, a transition of 2 blocks is used to improve the FTBR of a H-plane SIW horn at $f_{F T B R}=17 \mathrm{GHz}$. The substrate used is Rogers TMM 4 ( $\left.h=1.524 \mathrm{~mm}, \varepsilon_{r}=4.5\right)$ and the horn dimensions are $[\mathrm{mm}]$ : $W=30, D=70, a=7$.

The first step is to find the strip length $L$ that allows to maximize the FTBR. To do so, a value for $\varepsilon_{r p p}$ of 3.83 is found with the quasi-static approximation of [16] and a length $L=3 \mathrm{~mm}$ is obtained using (4).

Secondly, full-wave simulations are needed to find the right value of $s_{1}$ that makes $A_{1} \simeq A_{2}$. Notice that there will be always a difference between $A_{1}$ and $A_{2}$ due to radiation losses and, therefore, the back radiation can be greatly attenuated but not completely canceled. Using a reasonable $15 \%$ difference between both amplitudes (similarly to the radiation losses of a microstrip gap discontinuity [17]) a FTBR of $20.6 \mathrm{~dB}$ is predicted with (2).

To illustrate the effect and importance of the gap $s_{1}$, the simulated H-plane radiation patterns of different SIW horn configurations are shown in Fig. 4. In particular, a conventional SIW horn and a 2 block transition SIW horn $\left(L=3 \mathrm{~mm}, s_{0} \ll \lambda_{0}\right)$ with different $s_{1}(0.1 \mathrm{~mm}$ and $0.8 \mathrm{~mm})$ are compared. As expected, a poor FTBR is achieved when no transition is used $(0.8 \mathrm{~dB})$ and when $s_{1} \ll \lambda_{0}$, i.e., equal to $0.1 \mathrm{~mm}(4.6 \mathrm{~dB})$. But for an optimized $s_{1}$ value of $0.8 \mathrm{~mm}$, an excellent FTBR of $19.2 \mathrm{~dB}$ is obtained in accordance with the predictions of our approximate array model.

The described procedure allows therefore to design the transition $\left(L\right.$ and $\left.s_{1}\right)$ in order to obtain a high FTBR. Of course, this procedure will be of real interest provided that the SIW horn can be well matched for the same transition and at the same frequency. This important point is investigated in Section IV.

\section{SimultaneOUS FTBR AND MATCHING IMPROVEMENT}

The conditions to achieve simultaneously a good $\left|S_{11}\right|$ and a good FTBR are in general not compatible. This means that the frequency for which the SIW horn is well matched, $f_{\text {match }}$, and 


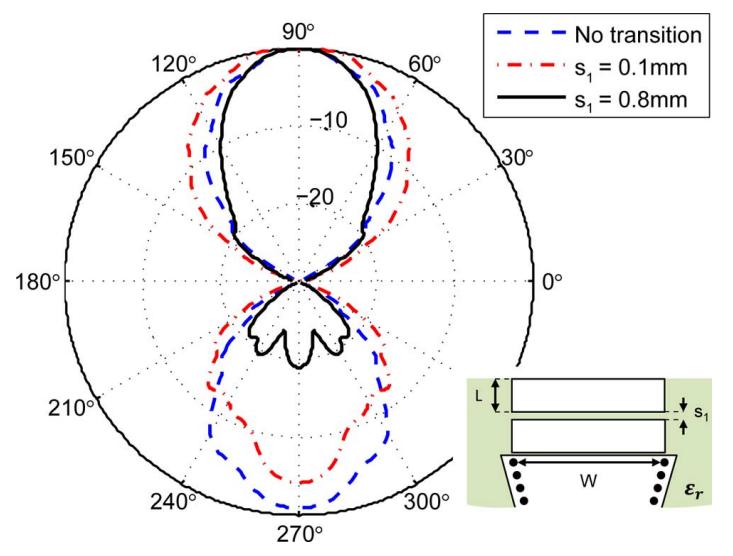

Fig. 4. Simulated normalized radiation pattern (H-plane) at $17 \mathrm{GHz}$ of a SIW horn with and without transition for different $s_{1}$ values. The SIW horn parameters are $W=30 \mathrm{~mm}, D=70 \mathrm{~mm}, a=7 \mathrm{~mm}$ and $\varepsilon_{r}=4.5$.

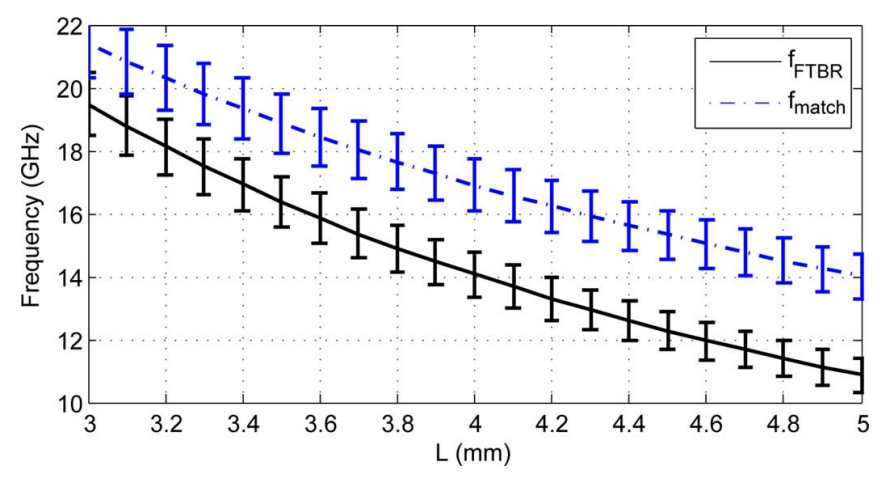

Fig. 5. $f_{F^{\prime} T B R}$ and $f_{\text {match }}$ as a function of $L$ of a SIW horn with a 2 block transition. The SIW horn parameters are $W=32.5 \mathrm{~mm}, D=35 \mathrm{~mm}, a=$ $8 \mathrm{~mm}$ and $\varepsilon_{r}=3.27$.

the one for which the FTBR is high, $f_{F T B R}$, are different. The formula to estimate $f_{\text {match }}$ is recalled in the Appendix.

To illustrate this lack of compatibility, let us consider the following practical example, where a substrate of $h=1.91 \mathrm{~mm}$ and $\varepsilon_{r}=3.27$ is used to implement a $15 \mathrm{GHz}$ SIW horn of dimensions [mm]: $W=32.5, D=35, a=8$. On one side, the condition to maximize the FTBR (4) gives an initial value for $L$ of $3.8 \mathrm{~mm}$. After full-wave simulations, the optimum values of $L=3.9 \mathrm{~mm}$ and $s_{1}=0.7 \mathrm{~mm}$ are found. On the other hand, by applying these dimensions in (11), a $f_{\text {match }}$ of $17.2 \mathrm{GHz}$ is predicted. To make $f_{\text {match }}=f_{F T B R}=15 \mathrm{GHz}$, a length $L$ of $4.55 \mathrm{~mm}$ would be required, but this length would deteriorate the FTBR below $10 \mathrm{~dB}$.

To confirm and quantify this lack of compatibility, the values of $f_{F T B R}(4)$ and $f_{\text {match }}(11)$ are plotted in Fig. 5 as a function of the strip length $L$. A $10 \%$ window around both frequencies is considered to take into account the bandwidth of the improvements. It is shown that, for a given transition, the frequency bands for $f_{F T B R}$ and $f_{\text {match }}$ do not overlap.

\section{A. Printed Transition With Gratings}

By analyzing the expressions in (4) and (11), it can be seen that the effective permittivity of the parallel plate waveguide $\varepsilon_{r p p}$ has a greater effect on the value of $f_{F T B R}$ than on that of

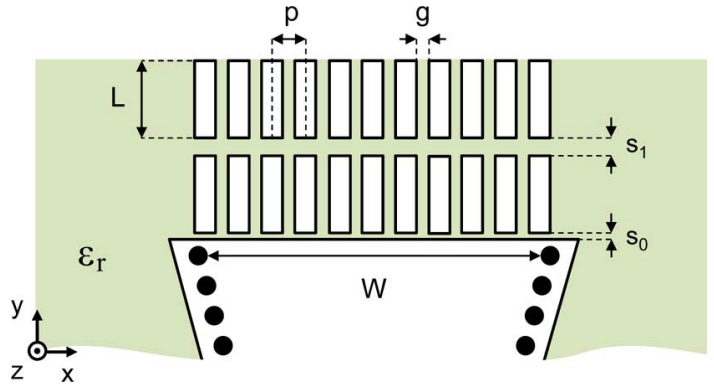

Fig. 6. Top view of a H-plane SIW horn with a 2 blocks rectangular grating transition with the associated notations.

$f_{\text {match }}$. Therefore, a way to simultaneously achieve both improvements, i.e., $f_{F T B R} \simeq f_{\text {match }}$, is to decrease the value of $\varepsilon_{r p p}$. To do so, the parallel plate structure composing each transition block must be modified. Instead of using full metallic strips of width $W$, a grating is introduced along the transversal direction $x$.

A possible grated transition with a rectangular pattern is shown in Fig. 6, where $p$ defines the grating periodicity and $g$ the separation between gratings. Obviously, as $g$ increases, the value of $\varepsilon_{r p p}$ decreases. However, the value of $g$ should remain reasonably small to avoid undesired radiation. Hence, it is recommended that $g<p / 2$ and $p<W / 10$.

The presented rectangular gratings are applied to the previous $15 \mathrm{GHz}$ SIW horn. Three versions of a 2 block transition with gaps of $s_{0}=0.2 \mathrm{~mm}$ and $s_{1}=0.7 \mathrm{~mm}$ are compared to show the advantages of the gratings:

- SIW horn A: Transition optimized for FTBR $(L=$ $3.9 \mathrm{~mm})$

- SIW horn B: Transition optimized for $\left|S_{11}\right|(L=$ $4.55 \mathrm{~mm})$

- SIW horn C: Transition with gratings optimized for both FTBR and $\left|S_{11}\right|(L=4.55 \mathrm{~mm}, p=2.6 \mathrm{~mm}, g=$ $1.2 \mathrm{~mm}$ )

The procedure to determine the values of $p$ and $g$ for SIW horn $\mathrm{C}$ consists in transforming gradually the full metallic strip into gratings. Thus, we decrease the effective permittivity $\varepsilon_{r p p}$ until reaching the desired value that makes $f_{F T B R} \simeq f_{\text {match }}$. Full wave simulations are needed to obtain the exact dimensions of $p$ and $g$.

The simulated radiation patterns and the $\left|S_{11}\right|$ are plotted in Fig. 7(a)-(b), respectively. As expected from (4), a high FTBR is achieved with horn $\mathrm{A}(18.6 \mathrm{~dB})$ and a low one with horn $\mathrm{B}$ (8 dB). Concerning the $\left|S_{11}\right|$, horn $\mathrm{B}$ is well matched at $15 \mathrm{GHz}$, but horn $\mathrm{A}$ is not. Horn $\mathrm{C}$ has the same strip length as horn $\mathrm{B}$ but gratings are introduced across the strips. As expected, the FTBR is greatly increased $(15.4 \mathrm{~dB})$ while the resonant frequency is slightly shifted but still well matched at $15 \mathrm{GHz}$. Therefore, the only horn which simultaneously presents a good $\left|S_{11}\right|$ and a good FTBR is the one with a grated transition, horn C.

\section{B. Discussion on the Gratings Shape}

In addition to the effect on the FTBR, the gratings also introduce other small changes in the radiation performances. Indeed, the metallic surface at the aperture is no more continuous and the amplitude distribution becomes less uniform. Hence, as it 


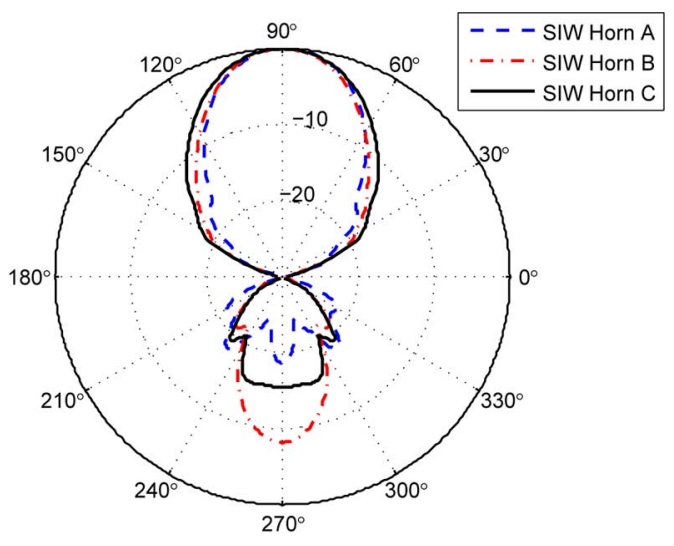

(a)

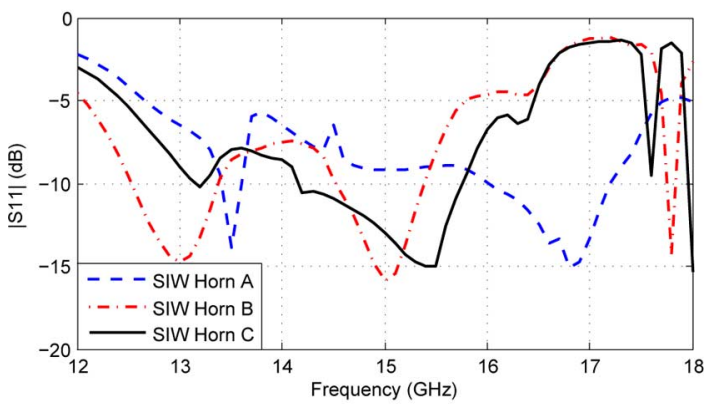

(b)

Fig. 7. Simulation results for the SIW horns A, B and C. (a) Normalized radiation patterns (H-plane) at $15 \mathrm{GHz}$. (b) Reflection coefficient.

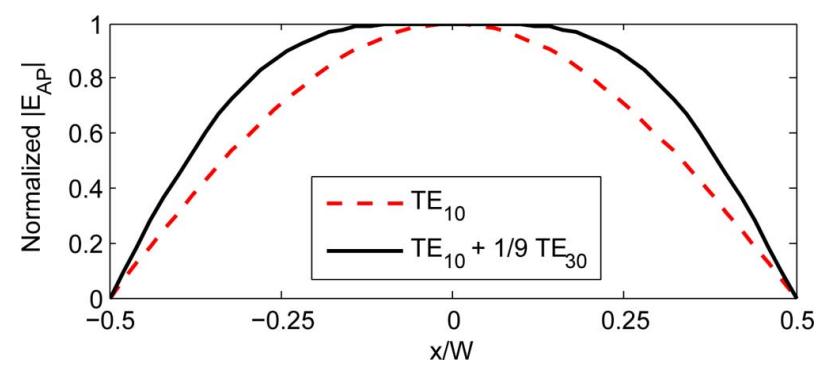

Fig. 8. Comparison between $\mathrm{TE}_{10}$ mode distribution and the distribution obtained with a proper combination of the $\mathrm{TE}_{10}$ and $\mathrm{TE}_{30}$ modes.

can be appreciated in Fig. 7(a), a slightly wider beamwidth is obtained when a grated transition is used. This effect could be controlled by using non-uniform gratings to further modify the field or current distribution.

The matching characteristics are also affected by the fact of not having a continuous metallic surface. In this case, the currents at the blocks' edges can follow slightly different paths, thus increasing the operational bandwidth. In Fig. 7(b), the horn with a grated transition, horn $\mathrm{C}$, achieves a $-10 \mathrm{~dB}$ bandwidth of $10.4 \%$ while exactly the same horn with a fully metallic transition, horn $\mathrm{B}$, yields only $6 \%$.

In order to further increase the bandwidth of this grated transition, the shape of the gratings can be tapered defining thus triangular gratings (see Fig. 1). Then, each block radiates along the taper instead of only at the edges of the parallel plates improving the matching as well as the FTBR bandwidth. For this reason, triangular gratings were chosen as the best option for the final manufactured prototype (see Section VI).

\section{Compact Horn Design}

A printed transition to improve both the $\left|S_{11}\right|$ and FTBR of SIW horn antennas has been presented in Sections III-IV. In this Section, the design of a SIW horn more compact than conventional ones is proposed. The aim is to properly shape the horn taper to reduce both the length $D$ and the aperture width $W$ for a given directivity. Such horn can then be easily implemented thanks to the flexibility provided by the SIW technology.

\section{A. Improvements in the Conventional Horn Performances}

The structure of a conventional horn usually includes a monomode waveguide before the beginning of the taper (see Fig. 3(a)). This waveguide is designed to completely attenuate the higher order modes, allowing only the propagation of the fundamental mode $\mathrm{TE}_{10}$. This determines the distribution of the electric field $E_{A P}$ at the horn aperture which is proportional to $\cos (\pi x / W)$. Then, if the cross sectional area increases with a linear flare angle along $x$, an spherical phase error $\delta(x)$ does appear [14].

In order to reduce the width of the horn aperture $W$, keeping the same directivity, the phase error $\delta(x)$ must be reduced and the field distribution $E_{A P}$ must become more uniform.

A common way to decrease $\delta(x)$ is to minimize the flare angle at the aperture. Thus, the horn radiates similarly to an openended waveguide which does not present spherical error. This concept is used, for instance, in the design of profiled horns [15].

A more uniform $E_{A P}$ can be generated by properly combining the $\mathrm{TE}_{10}$ and $\mathrm{TE}_{30}$ modes. In presence of both modes, the field distribution $E_{A P}$ is proportional to a linear combination of the two modes:

$$
E_{A P}(x) \propto \cos \left(\frac{\pi x}{W}\right)-\rho e^{j \psi} \cos \left(\frac{\pi 3 x}{W}\right)
$$

where $\rho$ defines the ratio between the amplitudes of the $\mathrm{TE}_{30}$ and the $\mathrm{TE}_{10}$ and $\psi$ their phase difference.

In order for the proposed $E_{A P}$ to be more uniform than a cosine function, it must strictly increase between $-W / 2$ and 0 and decrease between 0 and $W / 2$. This is obtained by applying the following operations:

$$
\begin{aligned}
& \frac{\partial E_{A P}(x)}{\partial x} \geq 0, \text { for }-\frac{W}{2} \leq x \leq 0 \\
& \frac{\partial E_{A P}(x)}{\partial x} \leq 0, \text { for } 0 \geq x \geq \frac{W}{2}
\end{aligned}
$$

which provide the optimum values $\rho=1 / 9$ and $\psi=0$. It is clearly shown in Fig. 8 that the combination $\mathrm{TE}_{10}+1 / 9 \mathrm{TE}_{30}$ generates a more uniform distribution than the $\mathrm{TE}_{10}$ alone.

\section{B. Proposed Horn Shape}

Several aspects must be considered in practice to fulfill the previous conditions for $E_{A P}$ and $\delta(x)$. First, to excite both 


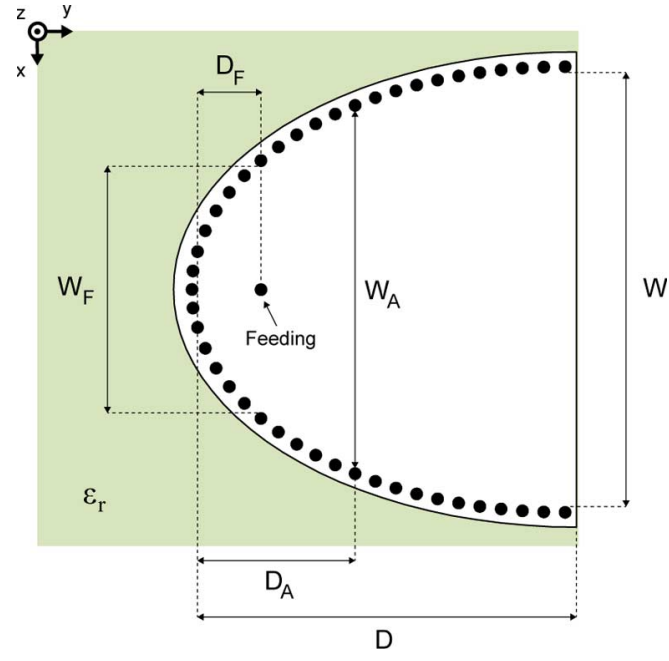

Fig. 9. Proposed superelliptical horn taper with the associated notations.

modes, $\mathrm{TE}_{10}$ and $\mathrm{TE}_{30}$, a coaxial probe or a multilayer transition should be used, rather than a monomode waveguide. Secondly, the taper should change smoothly to minimize reflections. A possible taper that satisfies these requirements is the one following a superelliptical function:

$$
\left(\frac{y}{D}\right)^{u}+\left(\frac{x}{\frac{W}{2}}\right)^{u}=1 \text {, with } u \geq 2 .
$$

The superelliptical function has been chosen since it inherently defines a $90^{\circ}$ angle with respect to the aperture which helps to minimize the flare angle thus decreasing $\delta(x)$. However, other shapes could provide satisfactory results provided that the procedure described in Section V-B1 is followed.

The proposed superelliptical horn is presented in Fig. 9. The aperture width $W$ is fixed by the desired beamwidth and the distance $D_{F}$ should be around $\lambda_{g} / 4$ for matching purposes, where $\lambda_{g}=\lambda_{0} / \sqrt{\varepsilon_{r}}$ is a guided wavelength. The taper width equal to the $\mathrm{TE}_{30}$ cut off wavelength is noted as $W_{A}$. Therefore, from the feeding point to $y=D_{A}$, the $\mathrm{TE}_{30}$ mode is attenuated defining the value of $\rho$. Then, from $y=D_{A}$ to $y=D$, the $\mathrm{TE}_{30}$ mode can propagate affecting the value of $\psi$.

To simultaneously fulfill the conditions of $\rho=1 / 9$ and $\psi=$ 0 , the taper shape can be modified by changing the parameters $D$ and $u$ in (8). A way to find these optimum values is to monitor the $\mathrm{TE}_{10}$ and $\mathrm{TE}_{30}$ modes between the feeding point and the horn aperture.

1) A Practical Design Example: For a horn of parameters $W=30 \mathrm{~mm}, D_{F}=3.07 \mathrm{~mm}$ and $\varepsilon_{r}=2.33$, the amplitude ratio $\rho$ and phase difference $\psi$ are plotted in Fig. 10 as a function of $D$ and for different values of $u$. It is shown that by changing $D$ and $u$, different combinations of $\rho$ and $\psi$ are obtained. In this case, the optimal values are $D=28.5 \mathrm{~mm}$ and $u=2$. P

To illustrate the importance of achieving the right $\rho$ and $\psi$ values, the electric field distribution inside two different horns is plotted in Fig. 11. The optimal case previously found is shown in Fig. 11(a) where the wavefronts along the horn present a shape

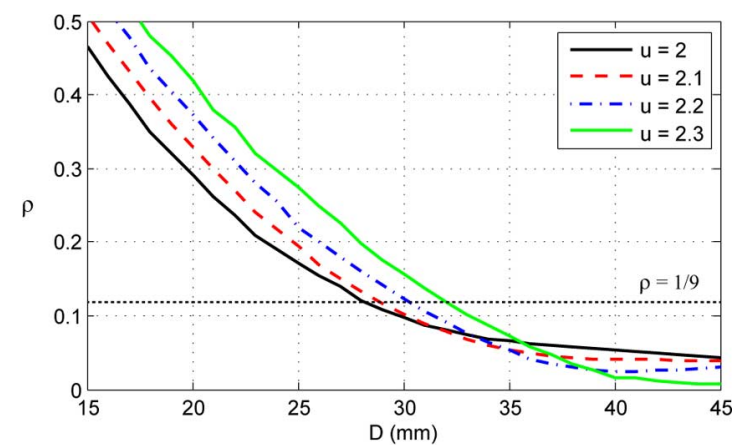

(a)

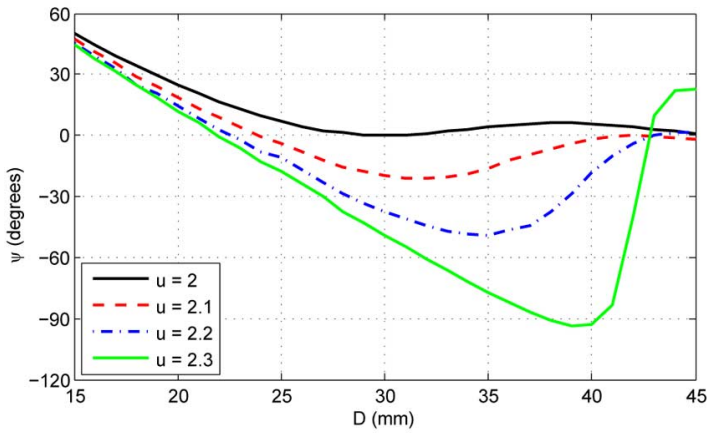

(b)

Fig. 10. Results at $16 \mathrm{GHz}$ for a superelliptical SIW horn with $W=30 \mathrm{~mm}$, $D_{F}=3.07 \mathrm{~mm}$ and $\varepsilon_{r}=2.33$. (a) Amplitude ratio $\rho$ and (b) phase difference $\psi$ as a function of $D$ and for different values of $u$.

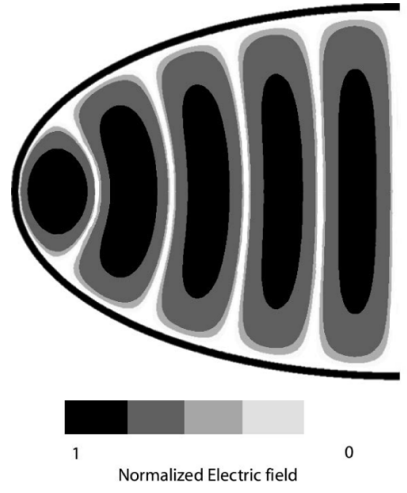

(a)

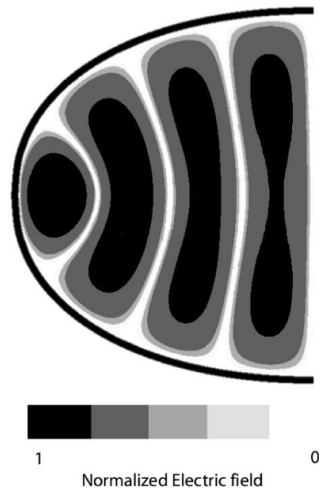

(b)
Fig. 11. Electric field distribution at $16 \mathrm{GHz}$ inside a superelliptical horn with $W=30 \mathrm{~mm}, D_{F}=3.07 \mathrm{~mm}$ and $\varepsilon_{r}=2.33$. (a) $D=28.5 \mathrm{~mm}$ and $u=2$. (b) $D=22 \mathrm{~mm}$ and $u=2.2$.

as in Fig. 8 and they are in phase. Let us now assume that $D$ is reduced while keeping the same $W$. In this case, in order to achieve $\psi \simeq 0$, the combination $D=22 \mathrm{~mm}$ and $u=2.2$ can be chosen (see Fig. 10). However, now $\rho$ equals 0.3 making the contribution of the $\mathrm{TE}_{30}$ stronger which creates two maximums in the wavefront front as shown in Fig. 11(b).

2) Discussion on the Proposed Shape: The strategy to successfully combine the $\mathrm{TE}_{10}$ and $\mathrm{TE}_{30}$ modes is conditioned by the aperture width $W$ and the substrate permittivity $\varepsilon_{r}$.

First of all, the dominant mode $\mathrm{TE}_{10}$ must not be attenuated along the taper. To ensure this, the taper width at the feeding 
position $W_{F}$ (see Fig. 9) must be wider than the cut off wavelength of the $\mathrm{TE}_{10}$ mode:

$$
\begin{gathered}
W_{F}=W \sqrt{1-\left(\frac{D-D_{F}}{D}\right)^{2}}>\frac{\lambda_{0}}{2 \sqrt{\varepsilon_{r}}} \\
W>\frac{\lambda_{0}}{2 \sqrt{\varepsilon_{r}} \sqrt{1-\left(\frac{D-D_{F}}{D}\right)^{2}}} .
\end{gathered}
$$

Secondly, to properly combine both modes, the $\mathrm{TE}_{30}$ mode must arrive at the aperture as a propagating mode. Hence, the aperture width $W$ must be wider than the cut off wavelength of the $\mathrm{TE}_{30}$ mode:

$$
W>\frac{3 \lambda_{0}}{2 \sqrt{\varepsilon_{r}}}
$$

By comparing (9) and (10), it can be seen that (10) is always the most restrictive condition for lengths $D$ shorter than $4.4 \lambda_{g}$, which includes most practical cases.

\section{Compact Horn vs. Conventional Horn}

In order to illustrate the compactness of the proposed horn with respect to a conventional one, let us see the size requirements for a given $\varepsilon_{r}$ as a function of the desired half power beamwidth $H P B W$. The parameters of the compact horn in Fig. 11(a) are $D=28.5 \mathrm{~mm}, W=30 \mathrm{~mm}$ and $\varepsilon_{r}=2.33$ generating a pattern with $\mathrm{HPBW}=37.5^{\circ}$.

To obtain the same radiation performances (HPBW = $37.5^{\circ}$ ) with a linear horn, a width $W=33 \mathrm{~mm}$ (instead of 30 $\mathrm{mm}$ for the compact horn) is required even when assuming that the phase error at the aperture $\delta(x)$ is negligible. Therefore, in this example, the fact of combining the $\mathrm{TE}_{10}$ and $\mathrm{TE}_{30}$ modes reduces the horn width in a $9 \%$.

When using a linear taper instead of a profiled one, the horn length must be considerably increased to also achieve a negligible $\delta(x)$ [15]. In this case, a linear horn of $D=54 \mathrm{~mm}$ is required being the compact horn $47 \%$ shorter.

To sum up, for the given HPBW and $\varepsilon_{r}$, the use of a superelliptical horn instead of a linear conventional horn halves the antenna surface ( $52 \%$ of surface reduction).

\section{Prototype Design AND Measurements}

A sectoral H-plane SIW horn antenna using the triangular grated transition and the superelliptical taper was built to validate the proposed concepts. Design steps to determine the SIW horn geometry are provided along with measurements.

\section{A. SIW Horn Design Steps}

A central frequency $f_{0}=14.8 \mathrm{GHz}$ (Ku-band) was selected for our prototype. The substrate used is a Rogers TMM3: $\varepsilon_{r}=$ 3.27 and $h=1.91 \mathrm{~mm}\left(<\lambda_{0} / 10\right)$. The working frequency band is defined by the following requirements: $\left|S_{11}\right|<-10 \mathrm{~dB}$, FTBR $>15 \mathrm{~dB}$ and H-plane HPBW $\simeq 45^{\circ}$. The steps to design the SIW horn are the following:
TABLE I

PARAMETERS OF THE SIW HoRN PROTOTYPE

\begin{tabular}{|c|c|c|c|c|c|c|c|}
\hline \multicolumn{3}{|c|}{ Compact Taper [mm] } & \multicolumn{5}{|c|}{ Triangular Grating Transition [mm] } \\
\hline \hline $\mathrm{W}$ & $\mathrm{D}$ & $\mathrm{u}$ & $\mathrm{L}$ & $\mathrm{s}_{0}$ & $s_{1}$ & $\mathrm{p}$ & $\mathrm{g}$ \\
\hline 24 & 20.45 & 2 & 4.8 & 0.2 & 0.2 & 2.2 & 0.15 \\
\hline
\end{tabular}

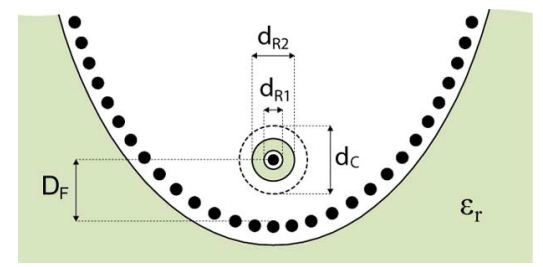

Fig. 12. Coaxial-to-SIW transition with the associated notations.

- Compact Taper

1) Given the HPBW, the horn aperture width $W$ is calculated assuming an $E_{A P}$ as in (5) with $\rho=1 / 9$ and $\psi=0$.

2) The conditions (9) and (10) are checked to verify that the proposed superelliptical taper can be applied for the given $\varepsilon_{r}$ and $W$. If not, a substrate with a higher permittivity must be chosen.

3) Knowing $W$, the procedure described in Section V-B is applied to determine the values for $D$ and $u$.

\section{- Printed Transition}

1) To simultaneously improve the FTBR and the $\left|S_{11}\right|$ at a given central frequency $f_{0}$, a printed transition of 2 blocks is used.

2) To obtain $f_{F T B R}=f_{0}$, the strip length $L$ is found using (4). Simulations are needed to find the right value for $s_{1}$ as well as to optimize the value of $L$.

3) The values of $L$ and $s_{1}$ are applied in (11) to check if also $f_{\text {match }}=f_{0}$.

4) If $f_{\text {match }} \neq f_{F T B R}=f_{0}$, the grating strategy presented in Section IV-A is implemented.

After following these design steps, the geometrical parameters fully defining our antenna (Fig. 1) are given in Table I. According to [1], the separation between via holes was chosen to be $1.1 \mathrm{~mm}$ for a hole diameter of $0.6 \mathrm{~mm}$ in order to keep the SIW radiation losses reasonably low.

\section{B. Feeding System}

A coaxial probe placed at the beginning of the taper is used to feed the antenna (Fig. 12). A coaxial-to-SIW transition with electric coupling is chosen since, in the case of thin substrates, this type of transition offers better performances than the magnetic one [18]. Thus, a ring-slot of width $\left(d_{R 2}-d_{R 1}\right) / 2$ is inserted between the current probe and the top copper layer. The circle of diameter $d_{C}$ denotes the opening at the bottom copper layer where the connector is placed.

In this feeding structure, the dimensions $d_{R 1}, d_{R 2}$ and $d_{C}$ strongly depend on the type of connector. In our case, the connector used is a 22 SMA-50-0-6/111NE and, after an optimization process, the following values are found $[\mathrm{mm}]: d_{R 1}=0.8$, $d_{R 2}=2, d_{C}=3.6, D_{F}=3.35$. 


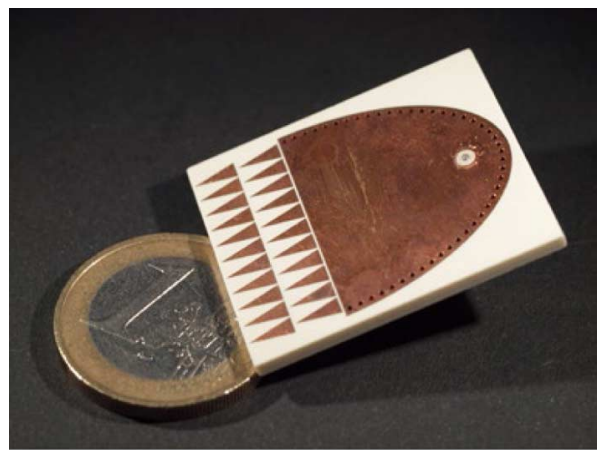

(a)

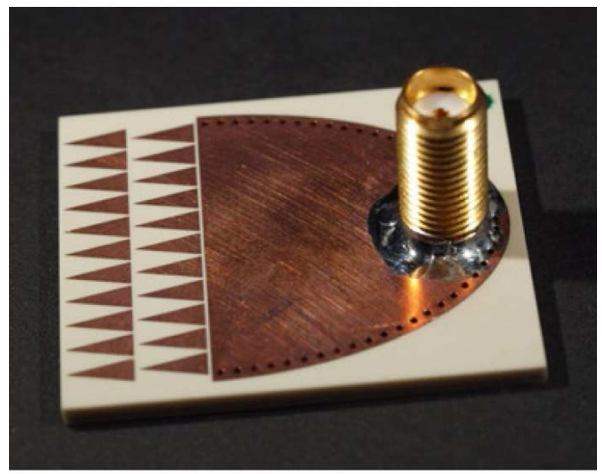

(b)

Fig. 13. (a) Top and (b) bottom view of the manufactured prototype of a SIW horn antenna with a triangular grated transition and a superelliptical taper.

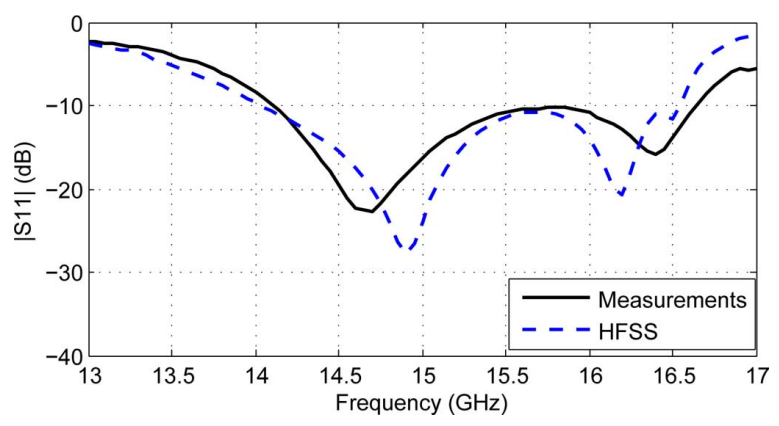

Fig. 14. Simulated and measured $\left|S_{11}\right|$ of the manufactured prototype.

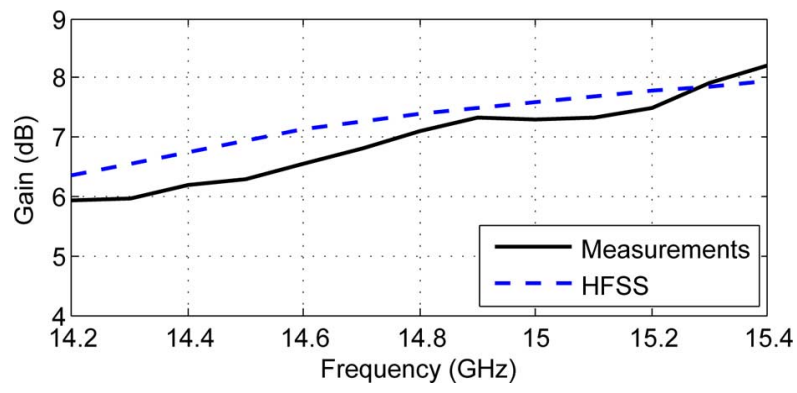

Fig. 15. Simulated and measured gain of the SIW horn prototype.

\section{Measurements}

The manufactured prototype is shown in Fig. 13. Its overall dimensions are $\left[\lambda_{0}\right]: 1.56 \times 1.26 \times 0.094$. The $\mathrm{H}$ - and E- plane radiation patterns for different frequencies are shown in Figs. 16 and 17. Full-wave simulations and measurements are in good

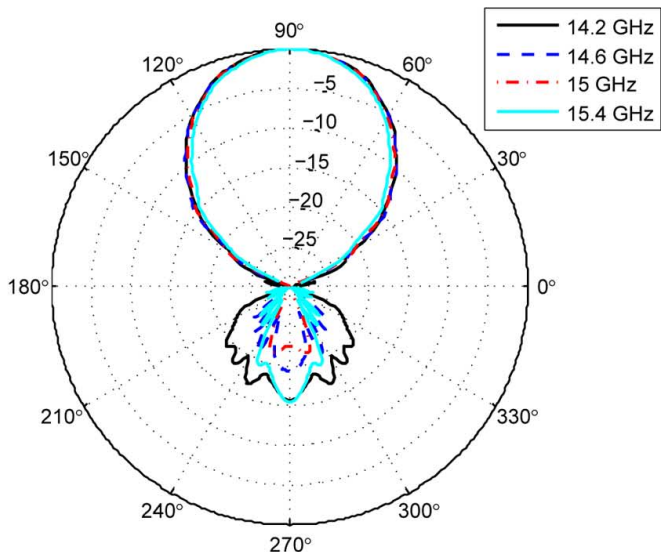

(a)

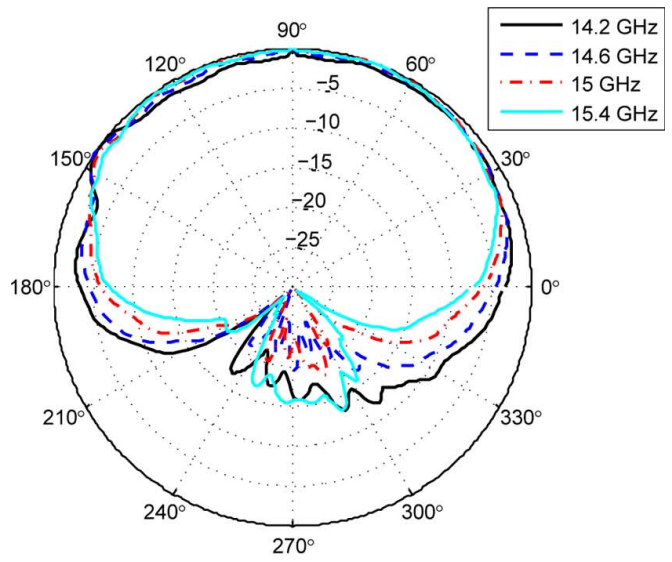

(b)

Fig. 16. (a) H-plane and (b) E-plane simulated radiation patterns of the SIW horn prototype.

agreement and a FTBR above $15 \mathrm{~dB}$ is achieved between 14.2 and 15.4 GHz. Low levels of the cross-polarization component below $-20 \mathrm{~dB}$ are also obtained.

At the same frequency range where the FTBR is greatly improved (14.2-15.4 GHz), a matching better than $-10 \mathrm{~dB}$ is also obtained (see Fig. 14). Furthermore, the $-10 \mathrm{~dB}$ bandwidth of the antenna extends from 14.1 to $16.6 \mathrm{GHz}$ defining a relative $16 \%$ bandwidth.

The addition of the proposed transition does not affect the typical behavior of horn antennas in terms of beamwidth. Indeed, the HPBW of the manufactured prototype only slightly changes from $46^{\circ}$ to $43^{\circ}$ as the electrical length of the aperture increases with the frequency. The simulated and measured gain of the antenna as a function of the frequency is plotted in Fig. 15 showing a measured gain at $f_{0}$ of $7.1 \mathrm{~dB}$.

\section{CONCLUSIONS}

The SIW technology offers very interesting possibilities to build H-plane horn antennas. However, the thickness of the SIW substrate is limited both by the reduced availability of thick substrates and by practical constraints in the technologies used to drill metallized via-holes. In general, good SIW performances call for substrates of at least $\lambda_{0} / 6$ thick and, with the current available substrates, this puts around $30 \mathrm{GHz}$ the lower frequency where SIW horns can be properly used. When SIW 


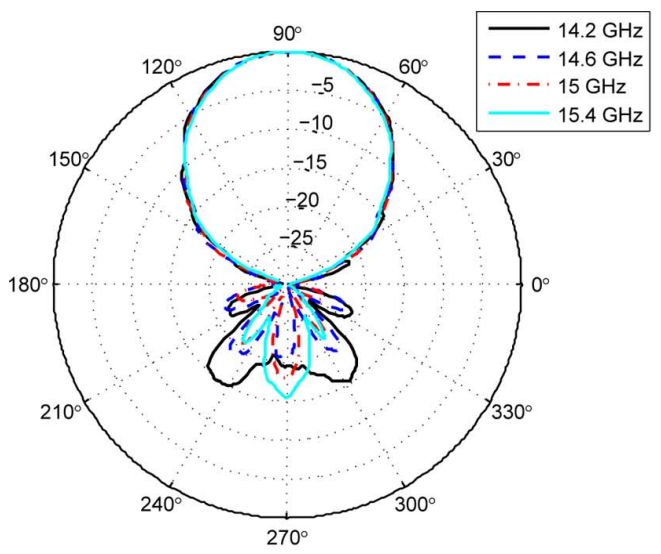

(a)

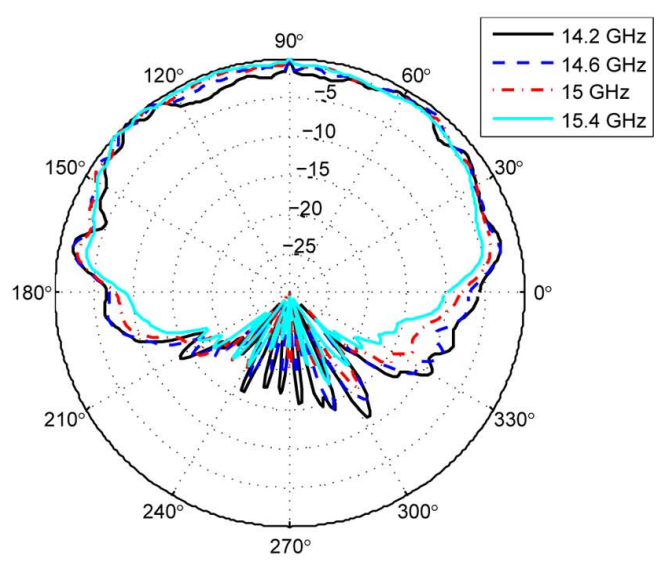

(b)

Fig. 17. (a) H-plane and (b) E-plane measured radiation patterns of the SIW horn prototype.

horns are built in thinner substrates, not only a strong mismatch between the antenna aperture and the air appears, but also the resulting antenna behaves like a slot antenna with a FTBR ratio near to unity.

This paper solves simultaneously both problems by introducing an innovative structure, which can be seen as a metallic grating integrated in the same SIW substrate as the horn antenna. By modelling this grating structure with a combination of transmission line and array theories, simple analytical design rules can be developed to fully characterize the needed geometry and to reasonably predict the antenna electromagnetic performances. The proposed antenna is further optimized by reducing its required dimensions for a given directivity. This is obtained by cleverly modifying the horn profile and by a departure from the traditional SIW waveguide terminated in a linear flared horn. Here, the inherent flexibility of SIW technology is used to develop a superelliptical taper for the horn. When combined with a coaxial excitation, a proper combination of TE modes is excited, leading to a more uniform distribution of fields in the SIW horn aperture and thus to unrivalled directivity vs. size properties.

The whole combination of improvements opens the door to the use of SIW horns at Ku-band and below. As a conclusive demonstration, a prototype has been designed following the guidelines developed in this paper and built using standard substrates and technologies. The novel SIW horn has transverse dimensions of $1.56 \lambda_{0} \times 1.26 \lambda_{0}$ and is thinner than $\lambda_{0} / 10$. Despite this miniaturization, it exhibits excellent electromagnetic properties. The matching is better than $-10 \mathrm{~dB}$ in the design frequency band $(14.2-15.4 \mathrm{GHz})$ and it can even be extended to a relative $16 \%$ bandwidth. The radiation pattern remains reasonably constant over the whole band with a half power beamwidth of $45^{\circ}$ and a FTBR better than $15 \mathrm{~dB}$.

In summary, the excellent performances of this prototype, together with its simple and low-cost construction, pave the way for a new family of SIW horns with a working range extended towards lower frequencies.

\section{APPENDIX}

The resonant frequency $f_{\text {match }}$ of SIW horn antenna with a 2 block transition is estimated by [13]:

$$
f_{\text {match }}=\frac{c}{2 L\left(1+\frac{0.7 h}{L}\right) \sqrt{\varepsilon_{r}} \sqrt{1-k_{2}\left(h, L, \varepsilon_{r}, s_{1}\right)}}
$$

where $k_{2}$ is a coupling factor given in [13]. Typically, a maximum $-10 \mathrm{~dB}$ bandwidth of $10 \%$ is achieved with this type of transition.

\section{REFERENCES}

[1] M. Bozzi, A. Georgiadis, and K. Wu, "Review of substrate-integrated waveguide circuits and antennas," IET Microw., Antennas Propag., vol. 5, no. 8, pp. 909-920, Jun. 2011.

[2] Z.-C. Hao, W. Hong, J.-X. Chen, X.-P. Chen, and K. Wu, "Compact super-wide bandpass substrate integrated waveguide (SIW) filters," IEEE Trans. Microw. Theory Tech., vol. 53, no. 9, pp. 2968-2977, Sep. 2005.

[3] Y. J. Cheng, W. Hong, and K. Wu, "Millimeter-wave substrate integrated waveguide multibeam antenna based on the modified R-KR lens," IEEE MTT-S Int., pp. 703-706, Jun. 2008.

[4] F. Giuppi, A. Georgiadis, A. Collado, M. Bozzi, and L. Perregrini, "Tunable SIW cavity backed active antenna oscillator," Electron. Lett., vol. 46, no. 15, pp. 1053-1055, 2010.

[5] J. Liu, D. Jackson, and Y. Long, "Substrate integrated waveguide (SIW) leaky-wave antenna with transverse slots," IEEE Trans. Antennas Propag., vol. 60, no. 1, pp. 20-29, Jan. 2012.

[6] E. Arnieri, G. Amendola, L. Boccia, and G. Di Massa, "Substrate integrated radiating waveguides with a coaxial feed," presented at the 2 nd Eur. Conf. on Antennas and Propagation (EuCAP), Nov. 2007.

[7] J. Yu, Y. Jiang, X. Lin, Z. Zhu, and Y. Fan, “A four-way half-mode SIW power divider with improved impedance match," in Proc IEEE 4th Int. Symp. on Microwave, Antenna, Propagation, and EMC Technologies for Wireless Communications, Nov. 2011, pp. 306-308.

[8] Z. Li, X.-P. Chen, and K. Wu, "A surface mountable pyramidal horn antenna and transition to substrate integrated waveguide," in Proc. Int. Symp. on Signals, Systems and Electronics, Feb. 2007, pp. 607-610.

[9] W. Che, B. Fu, P. Yao, Y. L. Chow, and E. K. N. Yung, "A compact substrate integrated waveguide H-plane horn antenna with dielectric arc lens: Research articles," Int. J. RF Microw. Comput.-Aided Eng., vol. 17, pp. 473-479, Sep. 2007.

[10] H. Wang, D.-G. Fang, B. Zhang, and W.-Q. Che, "Dielectric loaded substrate integrated waveguide (SIW) H-plane horn antennas," IEEE Trans. Antennas Propag., vol. 58, no. 3, pp. 640-647, Mar. 2010.

[11] R. Suga, H. Nakano, Y. Hirachi, J. Hirokawa, and M. Ando, "Costeffective $60-\mathrm{GHz}$ antenna package with end-fire radiation for wireless file-transfer system," IEEE Trans. Microw. Theory Tech., vol. 58, no. 12, pp. 3989-3995, Dec. 2010.

[12] Y. J. Cheng and Y. Fan, "Millimeter-wave miniaturized substrate integrated multibeam antenna," IEEE Trans. Antennas Propag., vol. 59, no. 12, pp. 4840-4844, Dec. 2011.

[13] M. Esquius-Morote, B. Fuchs, J.-F. Zürcher, and J. R. Mosig, "A printed transition for matching improvement of SIW horn antennas," IEEE Trans. Antennas Propag., vol. 61, no. 4, pp. 1923-1930, 2013.

[14] C. A. Balanis, Antenna Theory-Analysis and Design. Hoboken, NJ, USA: Wiley, 2005. 
[15] A. Olver, P. Clarricoats, A. Kishk, and L. Shafai, Microwave Horns and Feeds. London, U.K.: IEE, 1994, ch. 9.5.8.

[16] P. Benedek and P. Silvester, "Capacitance of parallel rectangular plates separated by a dielectric sheet," IEEE Trans. Microw. Theory Tech., vol. 20, no. 8, pp. 504-510, Aug. 1972.

[17] T.-S. Horng, S.-C. Wu, H.-Y. Yang, and N. Alexopoulos, "A generalized method for distinguishing between radiation and surface-wave losses in microstrip discontinuities," IEEE Trans. Microw. Theory Tech., vol. 38, no. 12, pp. 1800-1807, Dec. 1990.

[18] K. Song, Y. Fan, and Y. Zhang, "Eight-way substrate integrated waveguide power divider with low insertion loss," IEEE Trans. Microw. Theory Tech., vol. 56, no. 6, pp. 1473-1477, Jun. 2008.

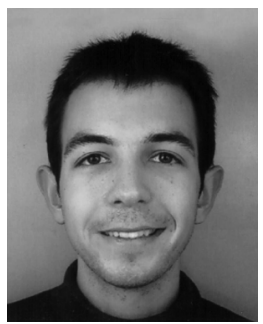

Marc Esquius-Morote (S'13) was born in Barcelona, Spain, in 1986. He received the degrees in telecommunication and electrical engineering both from the Technical University of Catalonia (UPC), in 2010 and 2011, respectively. He is currently working towards the Ph.D. degree at the Laboratoire d'Electromagnétisme et d'Acoustique (LEMA), Ecole Polytechnique Fédérale de Lausanne (EPFL), Switzerland.

During the last two years of his studies, he received a scholarship to work at the Antenna Lab group, UPC. His research interests are focused in the design and realization of substrate integrated structures and conformal systems.

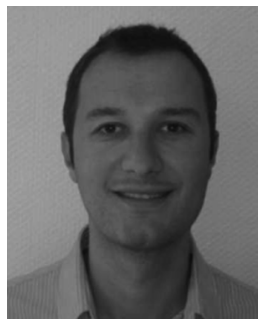

Benjamin Fuchs (S'06-M'08) received the Electronics Engineering degree and the M.S. degree in electronics from the National Institute of Applied Science (INSA) of Rennes, France, in 2004 and the Ph.D. degree from the University of Rennes 1 , France, in 2007.

In 2008, he was a Postdoctoral Research Fellow at the Swiss Federal Institute of Technology (EPFL), Lausanne, Switzerland. In 2009, he joined the Institute of Electronics and Telecommunications of Rennes (IETR) as a Researcher at the Centre National de la Recherche Scientifique (CNRS). Since 2011, he is on leave at Ecole Polytechnique Fédérale de Lausanne (EPFL), Switzerland. His research interests include millimeter-wave antennas, focusing devices (lens antennas) and array synthesis methods.

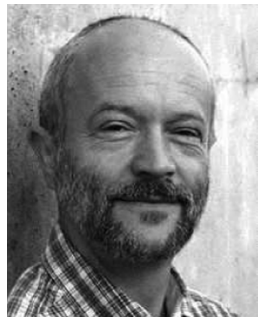

Jean-François Zürcher was born in Vevey, Switzerland, in 1951. He graduated with the degree of Electrical Engineer from Ecole Polytechnique Fédérale de Lausanne (Lausanne Institute of Technology), in 1974.

$\mathrm{He}$ is presently employed as permanent Scientific Associate with the Laboratoire d'Electromagnétisme et d'Acoustique EPFL, where he is the Manager of the Microwave Laboratory. His main interest lies in the domain of microstrip circuits and antennas. In 1988, he invented the SSFIP concept (Strip Slot Foam Inverted Patch Antenna), which became a commercial product. He developed instrumentation and techniques for the measurement of near fields of planar structures and microwave materials measurement and imaging. He is currently working on millimeter-wave antennas design and measurement.

$\mathrm{He}$ is the author or coauthor of more than 160 publications, chapters in books and papers presented at international conferences. He is one of the two authors of the book Broadband Patch Antennas (Artech, 1995). He holds nine patents.

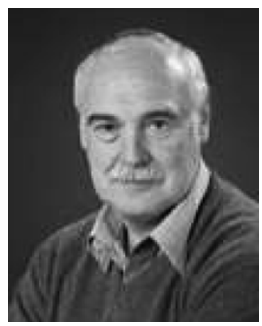

Juan R. Mosig (S'76-M'87-SM'94-F'99) was born in Cadiz, Spain. He received the Electrical Engineer degree in 1973 from Universidad Politécnica de Madrid, Spain, in 1973 and the Ph.D. degree from the Ecole Polytechnique Fédérale de Lausanne (EPFL), Switzerland, in 1983.

In 1976, he joined the Laboratory of Electromagnetics and Acoustics (LEMA), Ecole Polytechnique Fédérale de Lausanne (EPFL), Switzerland. Since 1991, he has been a Professor at EPFL and since 2000 the Head of the EPFL Laboratory of Electromagnetics and Acoustics (LEMA). In 1984, he was a Visiting Research Associate at Rochester Institute of Technology, Rochester, NY, USA. He has also held scientific appointments at University of Rennes (France), University of Nice (France), Technical University of Denmark, and University of Colorado at Boulder, CO, USA. His research interests include electromagnetic theory, numerical methods and planar antennas. He has authored four chapters in books on microstrip antennas and circuits and over 120 reviewed papers.

Dr. Mosig has been the Swiss Delegate for European COST Antenna Actions since the 1980's and the Chair for the two last COST Antenna Actions 284 and IC0603 ASSIST (2003-2011). He is also a founding member and General Chair of the European Association on Antennas and Propagation (EurAAP), owner of the EuCAP Conference series. He is the originator of a successful annual workshop, INTELECT, on Computational Electromagnetics. 